\title{
WRKY transcription factors: evolution, binding, and action
}

\author{
Xujun Chen ${ }^{1,2}$, Cheng $\mathrm{Li}^{1,2}$, Han Wang ${ }^{1,2}$ and Zejian Guo ${ }^{1,2,3^{*}}$ (i)
}

\begin{abstract}
The WRKY transcription factor (TF) gene family expanded greatly in the evolutionary process from green algae to flowering plants through whole genome, segmental, and tandem duplications. Genomic sequences from diverse plant species provide valuable information about the origin and evolution of WRKY domain-containing proteins. Accumulating data indicate that WRKY TFs bind W-box and/or other cis-elements, revealing the specificity of ciselement recognition based on the structure of the WRKY protein as well as the nucleotides adjacent to the core sequences. Physical interactions among WRKY proteins or between WRKY proteins and other regulatory factors afford important insights into the regulation of this TF family. WRKY proteins are essential players in the kinase signaling network. The interaction of plant resistance (R) proteins with WRKY TFs and the existence of unusual chimeric R-WRKY proteins suggest diversity in signaling pathways for rapid immune responses. In this review, we focus mainly on progress in understanding the function of WRKY TFs in response to biotic stresses and focus on their multiple roles in Arabidopsis and rice plants.
\end{abstract}

Keywords: Cis-element binding, Evolution, Metabolism, Plant immunity, Protein phosphorylation, WRKY transcription factor

\section{Background}

WRKY transcription factors (TFs) comprise a large gene family. Since the first report of the WRKY gene from sweet potato (Ishiguro and Nakamura 1994), numerous WRKY genes from a wide range of plant species have been characterized and shown to participate in growth, development, metabolism, and responses to environmental cues (Chi et al. 2013; Schluttenhofer and Yuan 2015; Phukan et al. 2016; Chen et al. 2017a; Jiang et al. 2017a). Accumulating data show that a large portion of WRKY genes are responsive to pathogens, elicitors, and defense-related phytohormones such as salicylic acid (SA) or jasmonic acid (JA), implying a major role for the WRKY gene family in plant immunity. WRKY TFs positively or negatively regulate various aspects of the plant innate immune system, which consists of pathogen-associated molecular pattern (PAMP)-triggered immunity (PTI) and effector-triggered immunity (ETI) (Jones and

\footnotetext{
* Correspondence: guozj@cau.edu.cn

${ }^{1}$ Key Laboratory of Pest Monitoring and Green Management, MOA, China

Agricultural University, Beijing 100193, China

${ }^{2}$ Department of Plant Pathology, China Agricultural University, Beijing

100193, China

Full list of author information is available at the end of the article
}

Dangl 2006). WRKY proteins participate in metabolism, including the synthesis of phytohormones, phytoalexins, and other defense-related chemicals (Wang et al. 2010; Schluttenhofer and Yuan 2015; Liang et al. 2017). WRKY TFs are also important players in the regulation of plant growth and development (Grunewald et al. 2012; Cai et al. 2014; Ding et al. 2015b; Li et al. 2015; Tian et al. 2017). In addition, WRKY proteins are involved in the plant response to abiotic stresses (Wang et al. 2007; Yokotani et al. 2013; Lee et al. 2018).

Increasing genome sequencing data from diverse plant species provide an excellent platform for the genomewide analysis of WRKY family genes. Modified hypotheses of WRKY gene evolution have been proposed based on genomic exploration and phylogenetic analyses (Rinerson et al. 2015). Chimeric WRKY TFs containing the WRKY domain (WD) and other novel protein domains have been discovered through genome mining (Rinerson et al. 2015; Mohanta et al. 2016). Genomic sequences have facilitated transcriptomic analyses of gene families, such as the WRKY genes, in a specific physiological process or stress condition and the discovery of important genes involved in particular circumstances

(c) The Author(s). 2019 Open Access This article is distributed under the terms of the Creative Commons Attribution 4.0 International License (http://creativecommons.org/licenses/by/4.0/), which permits unrestricted use, distribution, and reproduction in any medium, provided you give appropriate credit to the original author(s) and the source, provide a link to the Creative Commons license, and indicate if changes were made. The Creative Commons Public Domain Dedication waiver (http://creativecommons.org/publicdomain/zero/1.0/) applies to the data made available in this article, unless otherwise stated. 
(Ning et al. 2017; Yang et al. 2017; Xie et al. 2018). In addition, chromatin immunoprecipitation-sequencing (ChIP-seq) and DNA affinity purification sequencing (DAP-seq) have been used to identify genome-wide binding sites for TFs, such as the WRKY TFs (Liu et al. 2015; O'Malley et al. 2016; Birkenbihl et al. 2017). The integration of the available data may provide valuable information regarding the function and evolutionary relationships of orthologous genes.

Among the WRKY TFs, the formation of homo- and heterocomplexes to regulate gene expression through auto- and cross-regulatory mechanisms is often observed (Xu et al. 2006; Liu et al. 2016). WRKY proteins also interact with other proteins, such as receptors, kinases, and other TFs, and are interwoven in transcriptional regulatory networks (Ishihama et al. 2011; Chang et al. 2013; Xie et al. 2014; Sheikh et al. 2016; Lal et al. 2018). The formation of dynamic proteinprotein interactions is an important step in the undertaking of biological functions. In general, WRKY TFs regulate target genes through binding W-box (TTGA $\mathrm{CY}$, with the core sequence TGAC) cis-elements in gene promoters. However, some WRKY TFs recognize cis-elements without the $\mathrm{W}$-box core sequence. In this review, we summarize recent progress in understanding WRKY evolution and DNA binding activities and focus on WRKY protein interactions, especially those in response to biotic stress and the regulation of metabolism.

\section{WRKY structure and evolution}

The WRKY domain (WD) is approximately 60 amino acid residues in length and has potential DNA binding activity. The WD is derived from classical $\mathrm{C}_{2} \mathrm{H}_{2}$ zinc fingers present in mutator transposons by the addition of a WRKY-like motif upstream of the zinc finger (Babu et al. 2006). WRKY proteins are divided into three groups based on the type of zinc finger motif and the number of WDs (Eulgem et al. 2000). Group I and II members contain $\mathrm{C}_{2} \mathrm{H}_{2}$ zinc finger motifs, whereas WRKY proteins in group III have $\mathrm{C}_{2} \mathrm{HC}$ zinc fingers. WRKY genes are classified further into subgroups by phylogenetic analyses, especially group II, which is divided into at least five subgroups (IIa to IIe). A scheme of each WD subgroup in rice is shown in Fig. 1. Inconsistency beyond the two classification roles is observed. Reasons for this could be mutations in key amino acid residues, such as the very conserved heptad WRKYGQK and the amino acids involved in zinc binding; variations in zinc finger length; evolutionary intermediates between (sub)groups; and WD deletion. For example, OsWRKY5 and OsWRKY68, which are clustered in subgroup IIIa, contain $\mathrm{CX}_{7} \mathrm{CX}_{23} \mathrm{HXH}$ (X for any amino acid) zinc fingers that are similar to the subgroup IIIa $\mathrm{CX}_{7} \mathrm{CX}_{23} \mathrm{HXC}$ zinc finger, implying the possible divergence of $\mathrm{C}_{2} \mathrm{H}_{2}$ and $\mathrm{C}_{2} \mathrm{HC}$ zinc fingers (Wu et al. 2005). Wheat TaWRKY157 contains two WDs, but because it has a $\mathrm{C}_{2} \mathrm{HC}$ zinc finger, it is suggested to be an intermediate member of groups I and III (Ning et al. 2017). An extended zinc finger is found

\section{a I NT NWRKYGQK......RSYYKC-X ${ }_{4}-\mathrm{C}-\mathrm{X}_{22}-\mathrm{HNH}$ \\ I CT $\underline{\mathbf{R} W R K Y G Q K} \ldots . . . . R S Y Y K C-\mathrm{X}_{4}-\mathrm{C}-\mathrm{X}_{23}-\mathrm{HNH}$ \\ IIc RWRKYGQK.....RSYYRC-X ${ }_{4}-\mathrm{C}-\mathrm{X}_{23}-\mathrm{HXH}$ \\ IId SWRKYGQK......RGYYKC-X ${ }_{5}-\mathrm{C}-\mathrm{X}_{23}-\mathrm{HRH}$ \\ IIe AWRKYGQK......RGYYRC-X ${ }_{5}-\mathrm{C}-\mathrm{X}_{23}-\mathrm{HNH}$ \\ IIIa SWRKYGQK......RAYYRC-X ${ }_{7}-\mathrm{C}-\mathrm{X}_{23}$-HTC \\ IIIb QWRKYGQK......RSYYRC-X ${ }_{7}-\mathrm{C}-\mathrm{X}_{>23}-\mathrm{HTC}$ \\ IIa QWRKYGQK......C-X - -CPVKKKV-X $17-\mathrm{HNH}$ \\ IIb QWRKYGQK......C-X $-X_{5}$ CPVRKQV-X $17-\mathrm{HNH}$}

b

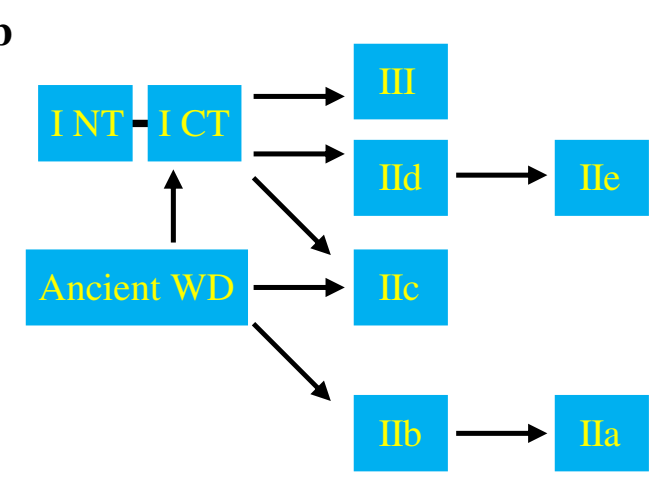

Fig. 1 Scheme of WRKY domain evolution. a Representative WRKY domain (WD) of each (sub)group. The consensus amino acid sequences of WDs from rice. The conserved zinc finger in red; the green $\mathrm{R}$ in bold for the conserved R-intron (phase 2), but the R-intron in Chlamydomonas reinhardtii at the position of underlined bold $\mathrm{R}$ (I CT line) instead; the light blue $\mathrm{V}$ in bold for the conserved $\mathrm{V}$-intron (phase 0 ) of subgroups Ila and Ilb. One of the introns in I NT of C. reinhardtii is at the position of underlined K (phase 2) in bold, however intronless for rice and Arabidopsis I NT WDs. b Hypothetical WD evolution. A hypothetical ancient WD existed as the ancestors of all WRKY genes. The ancient WD diversified to I CT/ IIc and IIb structures. The Ila and Ile WDs were deduced from IIb and Ile, respectively. The I NT WD is possibly gained by domain duplication and then subfunctionization. I NT and I CT for N- and C-terminal group I WD, respectively; arrows for the direction of evolution 
in rice, moso bamboo, and wheat group III WRKY proteins, i.e., $\mathrm{CX}_{6-7} \mathrm{CX}_{\mathrm{n}} \mathrm{HXC}$ with $n>23$ (Wu et al. 2005; Li et al. 2017; Ning et al. 2017). Members in group II have a $\mathrm{CX}_{5} \mathrm{CX}_{23} \mathrm{HXH}$ zinc finger motif, except subgroup IIc, which has a $\mathrm{CX}_{4} \mathrm{CX}_{23} \mathrm{HXH}$ zinc finger that is the same as that in the C-terminal WD (CTWD) of group I WRKY proteins. The subgroup IIc members can be clustered into separated clades, in which the ancient IIc clade neighbors the CTWD clade (Xu et al. 2016; Jiang et al. 2017b), suggesting a close evolutionary relationship between genes in group I and subgroup IIc. Subgroup IIc has also been proposed to be a separate group by Zhang and Wang (2005) or subgroup Ib by Wu et al. (2005). In addition to variations in zinc finger motifs, mutations in the consensus WRKYGQK sequence often appear in various plant species, and frequently observed variants include WRKYGKK and WRKYGEK (Wu et al. 2005; Yao et al. 2015; Jiang et al. 2017b; Yang et al. 2017; Song et al. 2018).

The diversification of WRKY genes has been explained by several hypotheses. Initially, group I WRKY genes were considered the ancestors of other WRKY genes, possibly due to the presence of only one group I WRKY gene in the unicellular green agar Chlamydomonas reinhardtii (Wu et al. 2005; Xie et al. 2005; Zhang and Wang 2005). Later, the available genome sequence of the filamentous terrestrial alga Klebsormidium flaccidum shows two WRKY genes, one in group I that contains two WDs and the other in subgroup IIb, reflecting the existence of a subgroup IIb gene early in the evolution of plants (Hori et al. 2014). Group III and group III-like genes are also found in the Selaginella moellendorffii and Physcomitrella patens mosses. However, subgroup IIa WRKY genes are absent in S. moellendorffii (Rinerson et al. 2015; Rensing et al. 2008). Therefore, Rinerson et al. (2015) proposed two alternative hypotheses of WRKY gene evolution: the "group I hypothesis" and the "IIa + IIb separate hypothesis". The "group I hypothesis" suggests that all WRKY genes evolved from group I CTWDs and that genes in subgroups IIa + IIb diversified ahead of the appearance of the conserved R-type (splicing at the codon of Arg, phase 2) intron present in the CTWDs and subgroups IIc, IId, and III. However, the IIa and IIb genes contain a V-type (phase 0) intron that is located before the Val residue in the WDs (Fig. 1). This difference in intron positions hints of divergence. The alternative "IIa + IIb separate hypothesis" suggests that genes in subgroups IIa and IIb evolved directly from a single WD gene in ancestral algae and that their lineage is different from that of the other group I WRKY genes (Rinerson et al. 2015). Furthermore, it seems reasonable to assume that an ancient, subgroup IIc-like WRKY gene is the ancestor of all WRKY genes, as proposed by Xu et al. (2016). The presence of nonplant WRKY genes in fungal, diplomonad, and amoebozoa species indicates lateral gene transfer events that occurred before the formation of WRKY groups in flowering plants and reflects ancient WRKY proteins that contained a single WD (Rinerson et al. 2015).

The WRKY gene family expanded greatly in the evolutionary process from one or a few genes in green algae to over 30 genes in the earliest land plant mosses and over 100 genes in some flowering plants (Fig. 2 and Additional file 1: Table S1). Whole genome, segmental, and tandem duplications were the main contributors to the expansion of the WRKY gene family. The amplification of group III WRKY genes in O. sativa ssp. japonica and $O$. nivara arose from segmental and tandem gene duplication (Wu et al. 2005; Xu et al. 2016). Group III WRKY genes are evolutionarily dynamic and play an important role in plant adaption. Phylogenetic analysis of group III WRKY proteins from ten representative monocots and dicots revealed monocot- and dicot-specific clades (Xie et al. 2018), supporting the hypothesis that the diversification of some WRKY genes occurred after the divergence of monocots and dicots (Wu et al. 2005). Nearly half of the TaWRKY genes arose from segmental duplication (Ning et al. 2017). The expansion of the groups I and II WRKY genes in Gossypium occurred through WGD (whole genome duplication) and tandem duplications (Ding et al. 2015a). The pineapple genome possesses relatively fewer WRKY genes than other monocots, which is possibly due to a lack of pangrass $\rho$ WGD events during pineapple evolution (Xie et al. 2018), while WGD and local gene duplication led to the existence of a large number of close GmWRKY homologs in soybean (Yang et al. 2017).

\section{WRKY binding elements}

Most WRKY proteins bind to W-box sequences in vitro, as determined by electrophoretic mobility shift assays (EMSAs). The solution structure of the AtWRKY4 CTWD complex with a W-box element was obtained by NMR (Yamasaki et al. 2012). It revealed that a fourstranded $\beta$-sheet enters the major groove of $\mathrm{B}$-form DNA in an atypical mode assigned to the $\beta$-wedge, in which the $\beta$-sheet is approximately perpendicular to the DNA helical axis. Residues in the conserved WRKYGQK sequence contact the DNA bases mainly by extensive apolar contacts with thymine methyl groups, which was verified by substitution of the thymine bases with uracil (Yamasaki et al. 2012). The structure of the WD-DNA complex can easily explain the results of previous mutational experiments and showed that Trp, Tyr, and two Lys residues in the WRKYGQK sequence are indispensable for DNA binding (Maeo et al. 2001; Duan et al. 2007; Ciolkowski et al. 2008). The zinc finger structures of WDs are also required for WRKY protein binding to the W-box element since the addition of zinc ion chelate eliminated the binding activity in EMSAs (Rushton et al. 1995). 


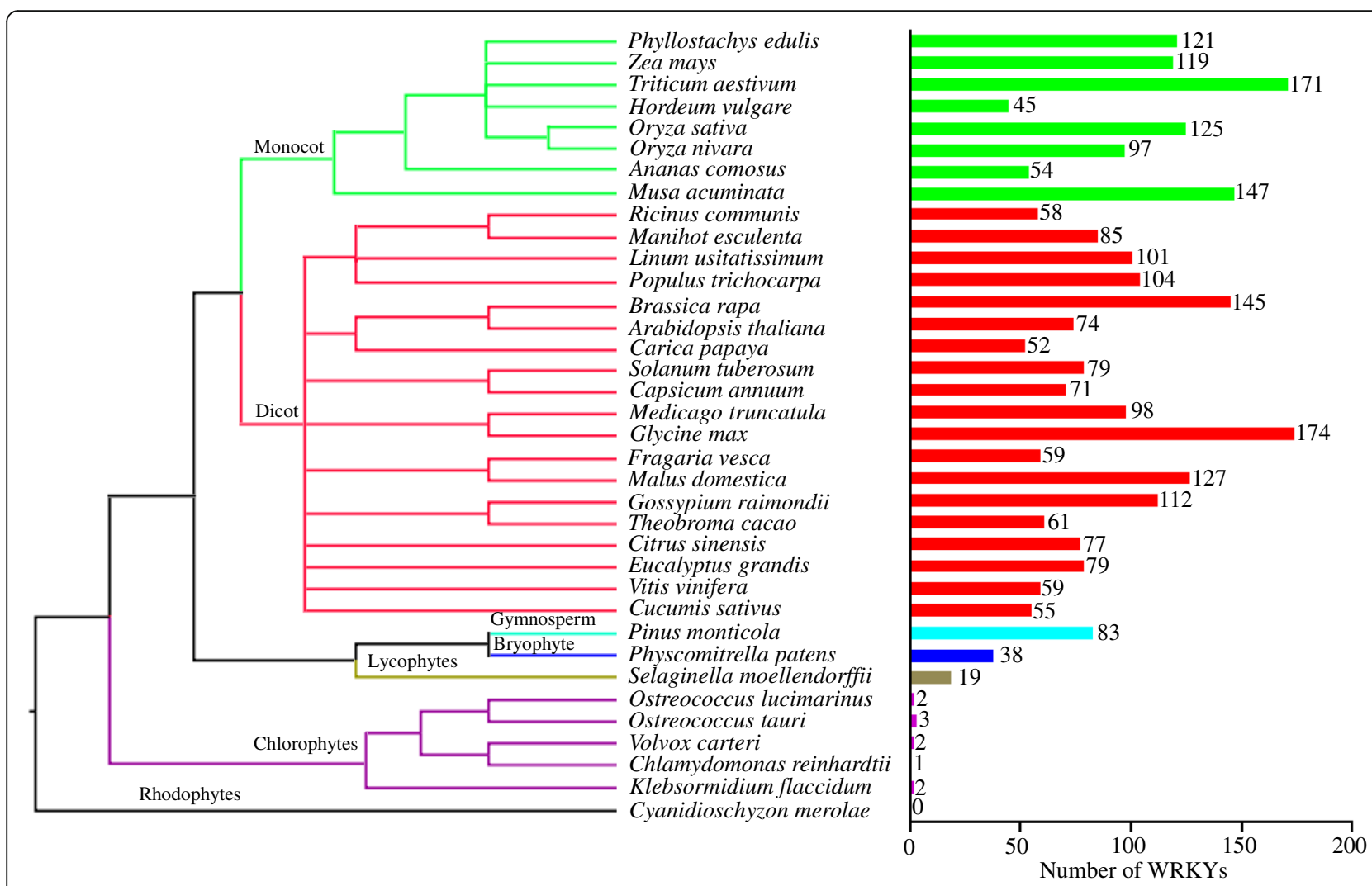

Fig. 2 Distribution of WRKY family genes in 36 plantae species. Phylogenetic tree was constructed based on NCBI taxonomy browser (https:// www.ncbi.nlm.nih.gov/Taxonomy/CommonTree/wwwcmt.cgi) and FigTree_v1.4.3 using Cyanidioschyzon merolae as an outgroup. The number of WRKYs on the right (for references see Additional file 1: Table S1). The expansion of WRKY family genes is mainly due to whole genome, segmental, and tandem duplications

Recently, the zinc ion was shown to bridge the dimerization of the WDs in the OsWRKY45 crystal structure (Cheng et al. 2019). The WD dimer interacts with the major groove of the W-box element, and water-mediated hydrogen bonds participate in the base-specific interaction between the protein and DNA. These results also explain why WRKY proteins often form homo- and heterocomplexes.

There have been several reports about the interaction of WRKY proteins harboring a WRKYGKK sequence with DNA cis-elements. Pepper CaWRKY1, a negative regulator of plant defense, bound to a DNA fragment with three TTGAC elements, as shown using EMSA analysis (Oh et al. 2008), whereas soybean GmWRKY6 and GmWRKY21 were unable to bind the W-box (Zhou et al. 2008). GmWRKY167, which contains a WD with a WRKYEDK sequence that is phylogenetically close to the N-terminal WD (NTWD) of group I WRKY proteins, was unable to bind the W-box element in vitro (Chi et al. 2015; Yang et al. 2017). It is unclear whether the inability of GmWRKY167 to bind the W-box was due to the change of the common GQK sequence to EDK in the WD close to the NTWD, since the CTWDs of group I WRKY proteins are mainly responsible for
DNA binding (Ishiguro and Nakamura 1994; de Pater et al. 1996; Maeo et al. 2001), its location in the Golgi, or both. Tobacco NtWRKY12, which also contains the WRKYGKK sequence, interacts with the WK-box (TTTTCCAC) but not with the consensus W-box. The WK-box was required for the induction of $P R-1 a$ expression by SA and bacterial elicitors, as shown in tobacco leaves (van Verk et al. 2008). Mutation of the GKK sequence to GQK or GEK abolished the binding activity of NtWRKY12 to both the WK- and W-boxes, and the GKK-containing AtWRKY51 recombinant protein did not bind to the WK- or W-boxes (van Verk et al. 2008). Rice OsWRKY7, which contains the WRKYGKK sequence, formed a complex with the W-box but not with the WK-box, and the mutation of GKK to GQK enhanced W-box binding activity, as examined by EMSA (Chen et al. unpublished data). In addition, WRKY proteins with the common WRKYGQK motif bind different types of cis-elements. OsWRKY13 bound to the W-box and PRE4 (TACTGCGCTTAGT), which are also present in its own promoter, both in vitro and in vivo (Xiao et al. 2013). Sugar signaling in barley2 (SUSIBA2, HvWRKY46) bound to both a sugar-responsive element (SURE, TAAAGATTA 
CTAATAGGAA) and the W-box element (Sun et al. 2003). AtWRKY70 bound not only to the classic W-box but also to the WT-box (YGACTTTT, GACTTT as the core sequence), which was essential to elicit the activation of gene expression in parsley protoplasts (Machens et al. 2014). Recently, the WD of AtWRKY50 was shown to interact with WT-box and WT-box-like elements, but the use of recombinant full-length AtWRKY50 hampered DNA binding (Hussain et al. 2018). The N-terminal region of AtWRKY50 possibly impedes DNA binding, as estimated from structure modeling. Additionally, AtWRKY50 interacted with the TGA2 and TGA5 TFs and simultaneously bound to proximal cis elements in the PR1 promoter; this then synergistically stimulated the SA-induced expression of PR1 (Hussain et al. 2018). These data suggest that the differences in amino acids among the WRKYGQK heptad and the rest of the WRKY proteins may affect the WD structure and lead to a preference for nucleotide bases, thereby enabling the selective and concerted regulation of many target genes by the WRKY TFs. In addition, nucleotides adjacent to the core sequence can also influence TF binding, as observed in the case of AtWRKY70 (Machens et al. 2014).

Molecular techniques have been developed to characterize regulatory elements and TF function on a genome-wide scale. The DAP-seq method interrogates genomic DNA with recombinant TF proteins for the discovery of TF binding sites (O'Malley et al. 2016). The ChIP-seq method was successfully used to identify AtWRKY33 binding sites in the Arabidopsis genome following infection with Botrytis cinerea and also revealed that AtWRKY33 negatively regulated abscisic acid (ABA) biosynthesis genes NCED3 and NCED5 in resistance towards this necrotrophic fungus (Liu et al. 2015). Birkenbihl et al. (2017) compared AtWRKY18, AtWRKY40, and AtWRKY33 binding sites in flg22, a 22 amino acid epitope of flagellin, triggered immunity, providing valuable insight into defining a genome-wide transcriptional regulatory network for defense responses involving WRKY TFs. The genome-wide analysis of WRKY binding sites also allows us to decipher its pleiotropic functions.

\section{WRKY shortcut immune signaling}

The recognition of strain-specific pathogen effectors by host resistance (R) proteins is linked with the serial activation of defense responses. Plant $\mathrm{R}$ proteins, termed NLRs, are comprised of N-terminal coiled-coil (CC) or Toll-interleukin 1 receptor (TIR) domains followed by nucleotide-binding and leucine-rich repeats. $\mathrm{R}$ proteins physically interact with TFs in plants. The barley mildew A $(M L A)$ locus encodes CC-type NLR (CNL) proteins, which mediates against the powdery mildew fungal pathogen Blumeria graminis (Shen et al. 2007). The CC domain of MLA10 interacts with two subgroup IIa
WRKY proteins, HvWRKY1 and HvWRKY2 (Shen et al. 2007). HvWRKY1 and HvWRKY2 function as repressors in basal defense, which is similar to the role of subgroup IIa WRKY family members in Arabidopsis. Later, the MLA10 CC domain was also shown to bind HvMYB6, which functions as a positive regulator in basal and MLA-triggered resistance to B. graminis (Chang et al. 2013). The activation of MLA10 was required to release HvMYB6 from the HvWRKY1 repressor complex and to stimulate the expression of HvMYB6-regulated genes. The data indicate that active MLA10 recruits HvMYB6 as an immediate activator for transcriptional reprogramming, which is sequestered by the HvWRKY1 repressor during the resting state (Chang et al. 2013). Panicle blast 1 (Pb1), which encodes a CNL protein, confers durable and broad-spectrum resistance to Magnaporthe oryzae races (Inoue et al. 2013). Pb1, through its CC domain, interacts with OsWRKY45 in the nucleus, and the interaction protects OsWRKY45 from ubiquitin proteasome system degradation. Conversely, Pb1-mediated blast resistance depends on OsWRKY45. A schematic diagram of the OsWRKY TFs involved in signaling networks is shown in Fig. 3a. XA21, a receptor kinase, is a pattern recognition receptor for Xanthomonas oryzae pv. oryzae (Xoo) resistance (Park and Ronald 2012). The XA21 intracellular region interacts with the subgroup IIa OsWRKY62 repressor exclusively in the nucleus of rice protoplasts. Intriguingly, XA21 is cleaved to release the intracellular kinase domain for nuclear translocation, which is required for XA21-mediated Xoo resistance. The XA21-mediated immune response through interaction with OsWRKY62 involves the derepression of defense (Peng et al. 2008; Park and Ronald 2012). OsWRKY46 and OsWRKY72 interact with the CNL-type BPH14 (brown planthopper resistance 14) protein ( $\mathrm{Hu}$ et al. 2017). The BPH14 interaction increases the stability of OsWRKY46 and OsWRKY72, which stimulates the expression of the receptor-like cytoplasmic kinase $(R L C K)$ gene and the callose synthase gene by binding to their promoters, providing an important clue for insect resistance mechanisms (Fig. 3a).

Arabidopsis Botrytis-induced kinase 1 (BIK1), a member of the RLCK family, functions downstream of the pattern recognition receptors (PRRs)-BAK1 complex (Veronese et al. 2006). EFR, the bacterial translation elongation factor $\mathrm{Tu}(\mathrm{EF}-\mathrm{Tu})$ receptor, phosphorylates BIK1 on a unique extended loop away from the core BIK1 kinase domain (Lal et al. 2018). Phosphomimetic mutants at these sites showed the increased accumulation of JA and SA and enhanced resistance to bacterial Pseudomonas syringae pv. tomato (Pst) DC3000. BIK1 is localized in the nucleus, where it interacts with AtWRKY33, AtWRKY50, and AtWRKY57. Interestingly, the BIK1 phosphomimetic mutant had significantly reduced trans-phosphorylation 

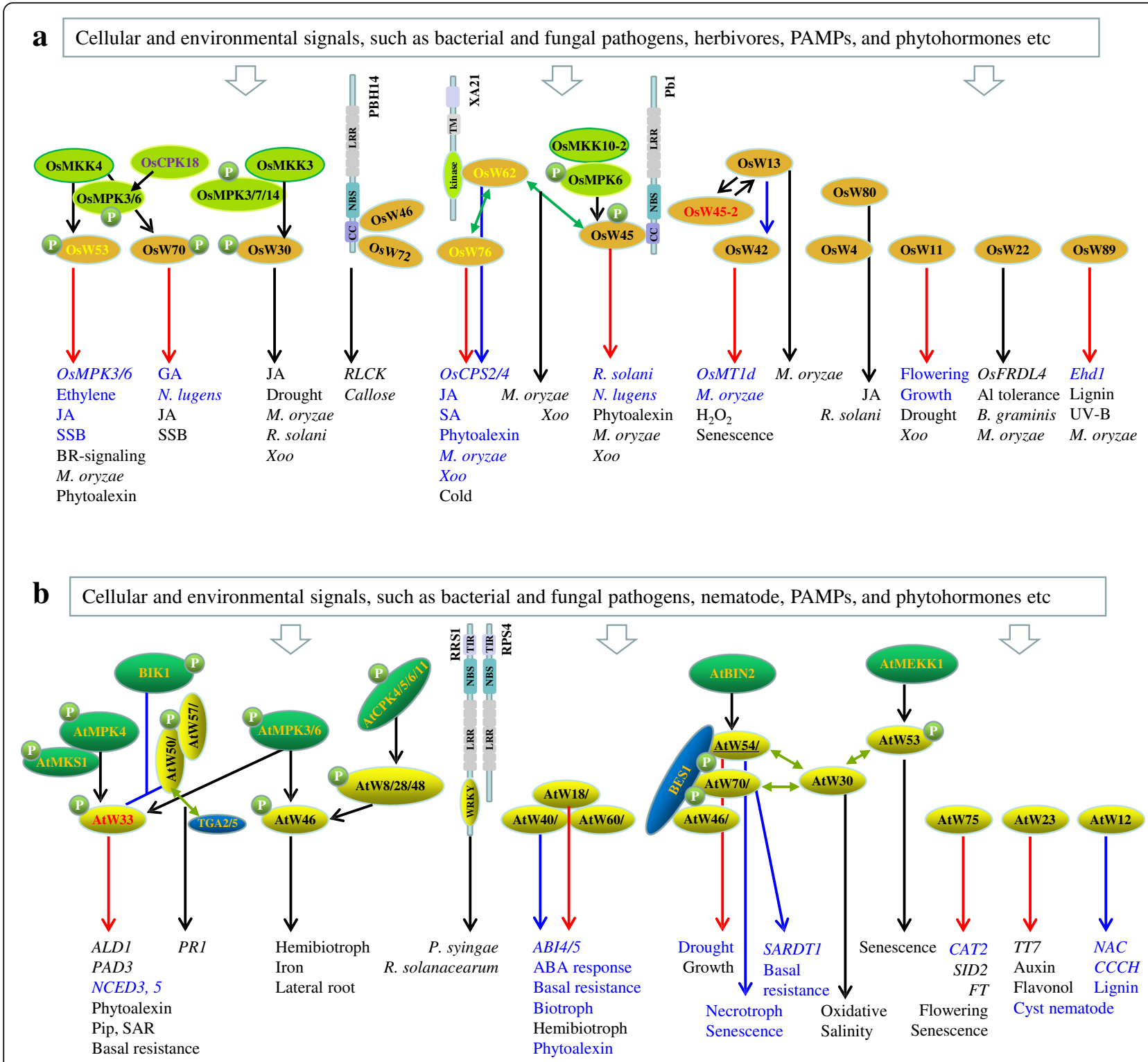

Fig. 3 Rice (a) and Arabidopsis (b) WRKY regulation in multiple pathways. OsWRKY and AtWRKY transcription factors (TFs) participate in response to various cellular and environmental stimuli. WRKY TFs may be activated by protein kinases and interaction with receptor-(like) proteins. Interaction among WRKY proteins or with other TFs, and the existence of WRKY cascade complicate WRKY regulatory network. For the details see the main text. Arrow in black for positive, blue for negative, and red for both positive and negative regulations; double arrow heads in green for interaction; WRKY in red for feedback positive and yellow for feedback negative regulations; word in blue for negatively regulated

activity towards AtWRKY33, AtWRKY50, and AtWRKY57 compared with that of native BIK1 (Lal et al. 2018). It has been proposed that the reduced trans-phosphorylation of AtWRKY50/AtWRKY57 and AtWRKY33 by pathogen-activated BIK1 derepresses the TF-mediated negative regulation of JA and SA induction, respectively (Lal et al. 2018). Figure $3 \mathrm{~b}$ shows the AtWRKY TFs that participate in signaling pathways.

Chimeric proteins consisting of domains typically found in both R proteins and WRKY TFs seem likely to exist in many plant species (Rinerson et al. 2015; Mohanta et al. 2016). AtWRKY52 is comprised of a TIR-type NLR structure and a C-terminal WD. The Sensitive to Low Humidity 1 (SLH1) gene was isolated as the $R$ gene RRS1-R (AtWRKY52-like) and confers resistance to the bacterial wilt pathogen Ralstonia solanacearum (Deslandes et al. 2002; Noutoshi et al. 2005). The $\operatorname{slh} 1$ mutant, caused by an amino acid insertion in the SLH1 WD that thereby impairs its W-box binding capacity, confers constitutive defense activation under conditions 
of low humidity, indicating that the WD functions negatively in SLH1 disease resistance (Noutoshi et al. 2005). The WD of RRS1-R is a "fused decoy" that intercepts pathogen effectors as they attempt to target WRKY TFs to inhibit immune transcription (Le Roux et al. 2015; Sarris et al. 2015). The $R$. solanacearum effector PopP2, which has acetyltransferase activity, interacts with RRS1-R in the plant cell nucleus and acetylates a Lys residue in the WRKYGQK motif to block WD binding to W-box elements. The head-to-head RRS1 and RPS4 pair, which both recognize the PopP2 and P. syringae pv. AvrRps4 effectors, have integrated a WD decoy to hijack effectors that target WRKY TFs (Le Roux et al. 2015; Sarris et al. 2015). Recently, Ma et al. (2018) showed that the WD in RRS1 interacts with its adjacent domain to maintain the RRS1-RPS4 NLR complex in an inactive state in the absence of effector. Effector AvrRps4 enables the interaction with the WD, thus disrupting the intramolecular association and derepressing the NLR complex, whereas PopP2-triggered activation occurs in a more complicated manner. Interactions of the NLR receptors with transcriptional networks and the presence of unusual NLR-WRKY proteins suggest the possibility of either diversity in signaling or accelerated signaling by shortcut signaling pathways for early, immediate immune responses against diverse invasion pathogens.

\section{WRKY interactions with kinases}

The mitogen-activated protein kinase (MPK) cascade is an important signaling event in plant defense against invading pathogens. MPKs are activated through phosphorylation by MPK kinases (MKKs or MEKs), which in turn are phosphorylated by upstream MPK kinase kinases (MEKKs). MPK3, MPK4, and MPK6 are often linked to plant responses to biotic and abiotic stresses. An increasing number of WRKY TFs have been identified as substrates of these MPKs and important components in MPK signaling pathways for the regulation of plant immunity and stress adaptation. In Nicotiana benthamiana, group I NbWRKY8 contains a D (docking) domain followed by an SP (serine/proline residue) cluster in the $\mathrm{N}$-terminal region, which is highly conserved in some group I WRKY proteins (Ishihama et al. 2011). The SP sites in NbWRKY8 can be phosphorylated by WIPK and SIPK (orthologs of AtMPK3 and AtMPK6, respectively) in vitro, and only two SP sites are phosphorylated in planta through the activation of NbMPKs by MEK2 (Ishihama et al. 2011). The phosphorylation of NbWRKY8 increases its binding to W-box elements and transactivation activity. The NbMPK-mediated phosphorylation of NbWRKY8 plays an important role in the activation of the immune response, as demonstrated by using phosphomimetic and virus-induced gene silencing mutants (Ishihama et al.
2011). Later, NbWRKY8 and its close homologues NbWRKY7, NbWRKY9, and NbWRKY11 were shown to bind with the promoter of $N b R B O H B$ via $\mathrm{MEK}^{\mathrm{DD}}$ and INF1 signal-responsive W-box containing elements to positively regulate $\mathrm{NbRBOHB}$ expression (Adachi et al. 2015). Furthermore, the authors demonstrated that the transactivation of $N b R B O H B$ through the MPK-WRKY route only occurred following a long-lasting and robust second ROS (reactive oxygen species) burst induced by ETI, but not a rapid ROS burst triggered by PTI (Adachi et al. 2015).

AtWRKY33, a close homologue of NbWRKY8, is required for the AtMPK3/AtMPK6- and pathogen-induced biosynthesis of camalexin, an indolic phytoalexin and phytohormone ethylene in Arabidopsis (Mao et al. 2011; Li et al. 2012). AtWRKY33 is phosphorylated by AtMPK3/ AtMPK6 in vitro and in vivo in response to $B$. cinerea infection, and the mutation of AtWRKY33 phosphorylation sites compromises its ability to fully complement the deficiency in camalexin accumulation in the atwrky33 mutant. Pathogen-inducible AtWRKY33 is regulated by the AtMPK3/AtMPK6 cascade in a potential positive feedback regulatory loop since AtWRKY33 targets its own promoter. AtWRKY33 is also present in the nuclear complex between AtMPK4 and AtMKS1, a substrate of AtMPK4 (Qiu et al. 2008). AtWRKY33 is released from the ternary complex upon challenge with $P$. syringae or flg22 treatment due to the phosphorylation of AtMKS1 by activated AtMPK4. Subsequently, released AtWRKY33 targets the promoter of PAD3 (phytoalexin deficient 3), which encodes the P450 enzyme CYP71B15, for the last step of camalexin biosynthesis (Fig. 3b). However, AtMPK4 is not needed for camalexin production in Arabidopsis in response to $B$. cinerea invasion (Mao et al. 2011). Nevertheless, these results indicate that AtWRKY33 functions downstream of AtMPKs in the regulation of camalexin biosynthesis. Pipecolic acid (Pip), a Lys catabolite, is key to establish systemic acquired resistance (SAR) in Arabidopsis upon localized pathogen infection. Pip is synthesized via ALD1 (AGD2-like defense response protein 1) and further converted by FMO1 (flavin-dependent monooxygenase 1) to $\mathrm{N}$-hydroxy-pipecolic acid, which is a vital component of SAR activation (Hartmann et al. 2018). Activated AtMPK3/AtMPK6 induces $A L D 1$ expression mediated by AtWRKY33 to elevate local Pip accumulation (Wang et al. 2018). In addition, Pip-induced AtMPK3/AtMPK6 activation or pathogen-triggered AtMPK activation is compromised in atwrky33 and ald 1 mutants. These results reveal that AtMPK3/AtMPK6 and AtWRKY33 together with ALD1 and Pip form a positive regulatory loop in SAR activation (Wang et al. 2018).

AtWRKY46, a group III WRKY protein member, was identified as a substrate of AtMPK3 by in vitro 
phosphorylation analyses (Sheikh et al. 2016). AtWRKY46 stability was reduced in protoplasts treated with flg22, whereas the mutation of either of the two phosphorylation sites to inactivate AtWRKY46 stabilized the protein. In a previous study, AtWRKY46 transcript levels were strongly upregulated in protoplasts and plants expressing the incompatible combination of RPM1 or PRS2 with avrRpm1, avrB or avrRpt2, and AtWRKY46 was an early marker gene in the convergent ETI signaling pathway (Gao et al. 2013). Furthermore, AtWRKY46 was synergistically regulated by WRKYs such as the subgroup IIc WRKYs AtWRKY8, AtWRKY28, and AtWRKY48 with the $\mathrm{Ca}^{2}$ ${ }^{+}$-dependent protein kinases (CPKs) CPK4, CPK5, CPK6, and CPK11. The CPKs phosphorylated AtWRKY8, AtWRKY28, and AtWRKY48, enhancing their binding activity to W-box elements. The AvrRPm1 and AvrRpt2 effectors triggered fast and long-lasting CPK activation but little AtMPK activation, unlike the induction of flagellin, suggesting the predominant role of CPKs in ETI signaling mediated by RPS2 and RPM1 in Arabidopsis (Gao et al. 2013). MPKs and CPKs also function in a parallel or synergistic manner in early innate immunity (Boudsocq et al. 2010). In addition, rice OsCPK18 interacts with OsMPK5 (equivalent to OsMPK3 in the text) and activates it through phosphorylation (Xie et al. 2014). The OsCPK18-OsMPK5 pathway negatively regulates rice resistance to $M$. oryzae and represents a direct link between calcium signaling and the MPK cascade.

The group I WRKY protein OsWRKY30 in rice interacts with multiple OsMPKs and is phosphorylated by OsMPK3, OsMPK7, and OsMPK14 (Shen et al. 2012). A phosphorylation-negative OsWRKY30 mutants at all SP sites did not exhibit improved drought tolerance in transgenic rice plants. In another study, the overexpression of OsWRKY3O conferred increased resistance to $M$. oryzae against sheath blight fungus Rhizoctonia solani (Peng et al. 2012). Enhanced disease resistance is associated with the upregulation of JA biosynthetic genes and increased JA production upon pathogen infection. Additionally, OsWRKY30 is a downstream target of the OsMKK3-OsMPK7 module and positively regulates the defense response against bacterial Xoo (Jalmi and Sinha 2016). Pathogen-inducible OsWRKY53 is a close homologue of NbWRKY8. The N-terminal conserved SP cluster in OsWRKY53 is the phosphorylation site for OsMPK3/OsMPK6, which is activated by OsMKK4 (Chujo et al. 2014). Phosphorylated OsWRKY53 has elevated transactivation activity, but there is no change in its ability to bind W-box elements. The overexpression of an OsWRKY53 phosphomimetic further enhanced resistance to a virulent $M$. oryzae strain and strongly upregulated numerous defense-related genes compared to their expression in plants overexpressing native OsWRKY53 (Chujo et al. 2014). OsWRKY53 is also rapidly induced by wounds and in a delayed fashion by attack from the striped stem borer (SSB) Chilo suppressalis (Hu et al. 2015). OsMPK3/OsMPK6 positively regulates OsWRKY53 transcription; however, OsWRKY53 negatively affects OsMPK3/OsMPK6 transcription and kinase activity after infestation with SSB larvae. Consequently, OsWRKY53 overexpressing plants accumulate less ethylene and jasmonate in response to SSB attack compared with the wild-type and RNAi lines and attenuate resistance to SSB larvae (Hu et al. 2015). These results suggest that OsWRKY53 acts as a negative feedback modulator of OsMPK3/OsMPK6 in defense against herbivores but activates disease resistance to blast fungus (Fig. 3a). It is curious that OsWRKY53 regulates OsMPK3/OsMPK6 in response to pathogen infection and has an antagonistic effect on a specific subset of genes in response to herbivore and pathogen invasion. Group I OsWRKY70 prioritizes defense over growth through increasing the biosynthesis of the defensive phytohormone JA and reducing the levels of the growth hormone GA upon attack by C. suppressalis ( $\mathrm{Li}$ et al. 2015). The activation of OsWRKY70 made rice plants more resistant to the chewing herbivore C. suppressalis but more susceptible to the rice planthopper Nilaparvata lugens, a piercing-sucking insect. Further experiments revealed that the increased JA level was associated with $C$. suppressalis resistance and that the reduced GA level was responsible for $N$. lugens susceptibility (Li et al. 2015). Recently, OsWRKY53 was identified as a positive regulator of rice brassinosteroid (BR) signaling that acts downstream of the OsMKK4-OsMPK6 cascade to control plant architecture (Tian et al. 2017). BRs promote OsWRKY53 protein levels and then negatively regulate their own expression. The trade-off between immunity and growth has important implications for the evolution and exploitation of the immune response to help protect crops from biotic stresses without growth penalty.

\section{WRKY complex and cascade}

Protein interactions have important implications in our understanding of the complicated network of functional interactions. Accumulating data show that WRKY proteins can form homo- and heterocomplexes. In Arabidopsis, AtWRKY18, AtWRKY40, and AtWRKY60, its three subgroup IIa members, interact by themselves and with each other through leucine zipper motifs (Xu et al. 2006). The three WRKY genes are negative regulators of basal defenses, acting in a complicated manner with additive and antagonistic effects (Xu et al. 2006). The additive effects of AtWRKY18, AtWRKY40, and AtWRKY60 are evident from their partially redundant roles in responses to biotrophic and hemibiotrophic pathogen infection based on the phenotypes of single, double, or triple 
mutants (Xu et al. 2006; Schön et al. 2013). In addition, the overexpression of AtWRKY18 increased resistance to $P$. syringae, while coexpression of AtWRKY18 with AtWRKY40 or AtWRKY60 made plants susceptible to the pathogen (Xu et al. 2006). The three WRKY genes also function in the ABA signaling pathway. ABA-induced AtWRKY60 transcription was strongly inhibited in the atwrky18 or atwrky 40 mutants, implying cooperation between AtWRKY18 and AtWRKY40 in the transcriptional activation of AtWRKY60 in response to ABA (Chen et al. 2010). AtWRKY40 is proposed to be linked to red light and ABA signaling and negatively mediate the ABAdependent inhibition of seed germination via downregulation of $A B I 5$ transcription (Geilen and Bohmer 2015). Physical interactions among AtWRKY18, AtWRKY40, and AtWRKY60 may alter their W-box binding capacity. AtWRKY60, which has little W-box binding activity alone, can enhance AtWRKY18 but reduce AtWRKY40 binding to W-box-containing sequences (Xu et al. 2006). The three WRKY proteins showed mostly antagonistic effects upon binding to some W-box elements from the $A B I 4$ and $A B I 5$ promoters that were tested (Liu et al. 2012).

WRKY gene transcription follows a sequential regulatory cascade. The group III WRKY protein OsWRKY45 is an important positive player in the resistance against the rice blast fungus $M$. oryzae (Shimono et al. 2007). OsWRKY13 in subgroup IIe and OsWRKY42 in subgroup IId are transcriptional repressors, and the overexpression of OsWRKY13 or the suppression of OsWRKY42 enhanced resistance to M. oryzae (Xiao et al. 2013; Cheng et al. 2015). OsWRKY42 functions downstream of OsWRKY13, which acts downstream of OsWRKY45-2, an allele of OsWRKY45/OsWRKY45-1 with a 10-amino acid difference from Minghui 63, an indica rice (Xiao et al. 2013; Cheng et al. 2015). OsWRKY13 and OsWRKY45-2 bind to each other and their own promoters, suggesting that OsWRKY13 and OsWRKY45-2 mutually control the transcription of each other's genes and are potentially fine-tuned by their own autoregulation. OsWRKY45-2-upregulated OsWRKY13 suppresses OsWRKY42; in turn, its protein derepresses JA-related genes in response to $M$. oryzae infection (Cheng et al. 2015). Additionally, transgenic rice plants overexpressing OsWRKY42 display an early senescence phenotype with an elevated hydrogen peroxide content, probably through the inhibition of OsMT1d gene expression (Han et al. 2014). OsWRKY62, a subgroup IIa WRKY member, has been analyzed as a repressor of disease resistance (Peng et al. 2008; Liu et al. 2016). Interestingly, OsWRKY62, after forming a heterocomplex with OsWRKY45, plays a positive role in the resistance to the rice blast and leaf blight pathogens, while the OsWRKY62 homocomplex acts as a repressor in the hypoxia response (Fukushima et al. 2016).
OsWRKY8O is induced by JA and the rice sheath blight fungus $R$. solani (Peng et al. 2016). Rice plants overexpressing OsWRKY80 exhibited enhanced resistance against $R$. solani concomitant with an increased level of OsWRKY4 transcription. Furthermore, OsWRKY80 specifically interacts with the promoter of OsWRKY4, which positively regulates the defense response to $R$. solani via the JA/ethylene-dependent signal pathway (Wang et al. 2015; Peng et al. 2016). The data suggest that OsWRKY80 together with OsWRKY4 functions as a positive regulatory circuit in disease resistance.

\section{WRKY regulates metabolites}

Upon pathogen infection, plants are triggered to produce active antimicrobial compounds termed phytoalexins, and their constitutive chemicals are named phytoanticipins (VanEtten et al. 1994). Such compounds are generally associated with the defense response, although each individual compound may have a greater or lesser effect against infectious pathogens. For example, camalexin and indole-glucosinolate in Arabidopsis and triterpenoid saponins in oats play an important role in plant defense against certain pathogens (Papadopoulou et al. 1999; Schön et al. 2013). Accumulating information has unraveled the regulatory networks and biosynthetic pathways of these bioactive compounds, including phytoalexins. The significance of WRKY TFs in the regulation of specialized metabolism has been reviewed by Schluttenhofer and Yuan (2015).

Rice plants infected with bacterial and fungal pathogens or treated by UV radiation produced diterpenoids, sakuranetin, phenolamides as phytoalexins (Cho and Lee 2015). Some of the compounds showed different activities against bacterial and fungal pathogens (Cho and Lee 2015). OsWRKY45 is required for benzothiadiazole (BTH) priming of diterpenoids such as momilactone $\mathrm{A}$ and phytocassanes $\mathrm{C}$ and $\mathrm{E}$ synthesis after $M$. oryzae inoculation (Akagi et al. 2014). OsWRKY62 and OsWRKY76, subgroup IIa members, form homo- and heterocomplexes and negatively regulate rice defense responses (Liu et al. 2016). Knockdown of OsWRKY62 and OsWRKY76 through RNA interference (dsOW62/76) led to the alternative splicing of the two genes and immunity to $M$. oryzae and Xoo infection. The individual knockout of OsWRKY62 or OsWRKY76 showed their role in a negative feedback regulation (Liu et al. 2016). Primary and secondary metabolism was reprogrammed in dsOW62/76 plants compared to metabolism in the wild-type plants (Liang et al. 2017). More importantly, dsOW62/76 plants accumulated a remarkable amount of diterpenoids, sakuranetin, and phenolamides, while the pool of free flavonoids was reduced. Additionally, the levels of SA and JA/ Ile-JA were increased in dsOW62/76 plants and individual 
OsWRKY62 and OsWRKY76 knockout lines compared to those in wild-type plants. In contrast, the overexpression of OsWRKY62 or OsWRKY76 repressed the accumulation of diterpenoids and sakuranetin (Yokotani et al. 2013; Liang et al. 2017).

The phytoalexin camalexin (3-thiazol-2'-yl-indole) has an important defense function against some necrotrophic, hemibiotrophic, and biotrophic pathogens, if not all of them (Ahuja et al. 2012). As mentioned earlier, phosphorylated AtWRKY33 regulates camalexin biosynthetic genes such as CYP71A13 and PAD3 (Qiu et al. 2008). AtWRKY18 and AtWRKY 40 are also implicated in phytoalexin biosynthesis. The double atwrky18 atwrky 40 mutant showed the transcriptional activation of genes related to camalexin and indole-glucosinolate biosynthesis, and the accumulation of these bioactive compounds was required for resistance towards the biotrophic powdery mildew fungus Golovinomyces orontii (Schön et al. 2013). AtWRKY12 in subgroup IIc acted in phenylpropanoid pathways via regulating lignin deposition (Wang et al. 2010). Plants expressing an atwrky12 mutant, like its ortholog in Medicago truncatula (mtstp), showed secondary wall thickening in pith cells and a significant increase in stem biomass. AtWRKY12 negatively regulates downstream NAC and CCCH-type zinc finger TFs that control lignin, xylan, and cellulose formation (Wang et al. 2010, Fig. 3b). Similarly, the suppression of AtWRKY12 orthologs in switchgrass and maize caused the ectopic deposition of secondary cell walls (Gallego-Giraldo et al. 2016). These results suggest that WRKY TFs involved in metabolism may have conserved roles across plant species (Schluttenhofer and Yuan 2015).

Jasmonate is often used as a key elicitor to regulate secondary metabolism. Jasmonate-responsive TFs, such as WRKYs and MYCs, are involved in the regulation of plant metabolism. The ectopic expression of AtWRKY18 and AtWRK40 together with AtMYC2 activated the MEP (2-C-methyl-D-erythritol 4-phosphate) pathway in Salvia sclarea hairy roots and consequently elevated the abietane diterpene content (Alfieri et al. 2018). Catharanthus roseus produces various terpenoid indole alkaloids that are of pharmaceutical importance. MeJA-inducible CrWRKY1, an ortholog of OsWRKY45, positively regulates the tryptophan decarboxylase gene through binding to its promoter, resulting in an increase in tryptamine followed by serpentine accumulation (Suttipanta et al. 2011). In the opium poppy (Papaver somniferum), PsWRKY binds to the tyrosine decarboxylase gene promoter to regulate benzylisoquinoline alkaloid production (Mishra et al. 2013). These decarboxylase-generated amines and phenolic acids in the phenylpropanoid pathway can form phenolamides, as observed in rice (Liang et al. 2017). Phenolic acid and polyamine conjugates also play a role in disease resistance. In wheat, TaWRKY70 imparts resistance against the Fusarium head blight pathogen Fusarium graminearum by regulating the synthesis of coumaroylagmatine and coumaroylputrescine (Kage et al. 2017). In another report, TaWRKY70 was positively involved in high-temperature wheat seedling resistance to the stripe rust fungus Puccinia striiformis f. sp. tritici (Wang et al. 2017).

\section{WRKY in multiple pathways}

Phytohormone BRs regulate multiple plant growth and developmental processes as well as their responses to environmental challenges. The BR signal may be transduced through a negative acting glycogen synthase kinase-3-like kinase BIN2 (BR-insensitive 2) to the BES/BZR1 (BR insensitive-EMS-suppressor-1/brassinazole-resistant 1 ) family TFs, which regulate the expression of BR-responsive genes. Three Arabidopsis group III WRKY TFs, AtWRKY46, AtWRKY54, and AtWRKY70, are induced by BRs and function redundantly in BR-regulated plant growth (Chen et al. 2017b). The three WRKYs interact with BES1 and cooperatively regulate BES1 target genes. The BES1 upstream regulator BIN2 can also interact with and phosphorylate AtWRKY46, AtWRKY54, and AtWRKY70, leading to the instability of at least AtWRKY54 in planta (Chen et al. 2017a, b). The atwrky46 atwrky 54 atwrky 70 triple mutant is deficient for BR-regulated plant growth but more tolerant to drought stress than wild-type plants, indicating that AtWRKY46, AtWRKY54, and AtWRKY70 play a positive role in plant growth and a negative role in drought responses (Chen et al. 2017b). In addition, AtWRKY46 is also involved in multiple processes including disease resistance, iron translocation under Fe deficiency, and lateral root development (Hu et al. 2012; Ding et al. 2015b; Yan et al. 2016).

The atwrky 54 atwrky 70 double mutant exhibits the increased accumulation of SA and $\mathrm{H}_{2} \mathrm{O}_{2}$ with accelerated leaf senescence and enhanced resistance against the necrotrophic pathogens $B$. cinerea and Pectobacterium carotovorum, suggesting that AtWRKY70 together with its closest homolog, AtWRKY54, negatively regulate senescence and resistance to necrotrophs (Besseau et al. 2012; Li et al. 2017). It is likely that the elevated SA content in the atwrky54 atwrky70 double mutant stimulates the appropriate accumulation of $\mathrm{H}_{2} \mathrm{O}_{2}$ and cell wall fortification, consequently raising resistance to necrotrophs but that this is not sufficient to increase resistance to biotrophs or hemibiotrophs (Li et al. 2017). AtWRKY30 interacts with two negative senescence regulators, AtWRKY54 and AtWRKY70, and one senescence activator, AtWRKY53, which was the first WRKY TF characterized as a senescence regulator. The expression of AtWRKY30, AtWRKY53, AtWRKY54, and AtWRKY7O is partially dependent on SA synthesis during senescence, while AtWRKY30 and AtWRKY53 but not AtWRKY54 
and AtWRKY7O are responsive to ROS such as $\mathrm{H}_{2} \mathrm{O}_{2}$ and ozone (Besseau et al. 2012). The $\beta$-glucuronidase activity driven by the AtWRKY30 promoter is induced strongly by chitin and especially cellobiose ( $\beta$-1,4-diglucose), which functions as a damage-associated molecular pattern molecule (Souza et al. 2017). Tolerance to oxidative and salinity stresses was increased by the constitutive expression of AtWRKY30 (Scarpeci et al. 2013). AtWRKY70 binds the W- and WT-box elements in the AtWRKY30 promoter, suggesting that AtWRKY70 may directly regulate AtWRKY30 expression (Machens et al. 2014) and not just their protein-protein interaction as mentioned above. Recently, AtWRKY70 was shown to repress SARD1 (SYSTEMIC ACQUIRED RESISTANCE $D E F I C I E N T$ 1) via direct binding to the WT-box in the promoter of $S A R D 1$, which encodes a positive regulator of plant defense that promotes SA biosynthesis (Zhou et al. 2018). AtWRKY70 is mainly an immune repressor in the absence of pathogens but activates immunity after pathogen attack, as revealed by transcriptomic analysis. The promoters for the genes with altered expression are enriched in WT-box elements. The authors suggested that the repression of SARD1 by AtWRKY70 contributes to fitness, because the WT-box motif is conserved within the Brassicaceae family (Zhou et al. 2018). AtMEKK1 regulates AtWRKY53, which is phosphorylated by the kinase and exhibits increased DNA binding activity (Miao et al. 2007). Interestingly, AtMEKK1 binds to the promoter of AtWRKY53, and the binding motif in AtMEKK1 is necessary for age-regulated AtWRKY53 expression (Miao et al. 2007). AtWRKY75 is a positive regulator of leaf senescence and flower timing (Guo et al. 2017; Zhang et al. 2018). AtWRKY75 binds to the promoter of the FLOWERING LOCUS T (FT) gene, whereas DELLA proteins interact with AtWRKY75 to attenuate its activation (Zhang et al. 2018). AtWRKY75 expression is induced by age, $\mathrm{SA}$, and $\mathrm{H}_{2} \mathrm{O}_{2}$. Meanwhile, AtWRKY75 promotes SA synthesis by upregulating SID2 (SA induction-deficient 2) transcription and suppressing CAT2 (catalase 2) transcription (Guo et al. 2017). The overexpression of AtWRKY75 accelerates leaf senescence, which can be compromised by silencing SID2 or increasing catalase activity. A tripartite amplification loop model involving AtWRKY75, SA, and ROS has been proposed, which provides insights into the molecular regulatory network of senescence (Guo et al. 2017). These results support the promoting role of SA and ROS in leaf senescence.

AtWRKY23, a subgroup IIc WRKY protein, participates in root development by controlling auxin distribution (Grunewald et al. 2012). AtWRKY23 regulates the synthesis of flavonols, particularly in the root tip, by stimulating the transcription of TT7 (transparent testa 7), which encodes flavonoid 3'-hydroxylase and mediates the conversion of dihydrokaempferol to dihydroquercetin (Grunewald et al. 2012). Consequently, the accumulation of quercetin, an endogenous negative regulator of auxin transport, is elevated in the roots of plants that constitutively express AtWRKY23 compared with its levels in the roots of wild-type plants. In an earlier study, AtWRKY23 was reported to be a plant-parasitic cyst nematode (Heterodera schachtii)-inducible gene (Grunewald et al. 2008). The expression of AtWRKY23 is stimulated during the early stages of nematode feeding site establishment, and the suppression of AtWRKY23 decreased susceptibility toward the parasitic nematode (Grunewald et al. 2008). Auxin-inducible AtWRKY23 expression is controlled by SOLITARY ROOT/INDOLE-3-ACETIC ACID14 (SLR/IAA14) as well as its downstream TFs AUXIN RESPONSE FACTOR (ARF) 7 and ARF19. Therefore, the AtWRKY23-mediated, flavonol-induced regulation of auxin transport for development could be hijacked by plant pathogens during evolution (Grunewald et al. 2012). A gain-of-function mutant, dlf1, of OsWRKY11, a very close homolog of AtWRKY23, exhibits semidwarf and late flowering phenotypes due to elevated OsWRKY11 transcription caused by a T-DNA insertion (Cai et al. 2014). In the dlf1 mutant, the alternative splicing of OsWRKY11 was observed along with the presence of a shorter transcript by a deletion in the third exon leading to premature translation. Plants overexpressing the short transcript showed a very high level of transgene transcription and a phenotype similar to that of the dlf1 mutant (Cai et al. 2014). The overexpression of wild-type OsWRKY11 decreased plant height but enhanced resistance to Xoo and tolerance to drought stress, while knock-down of OsWRKY11 compromised these phenotypes (Lee et al. 2018). These results indicate that OsWRKY11 positively regulates biotic and abiotic stress responses with a possible growth penalty. The transcription level of OsWRKY89 (renamed 104 by the CGSNL) was strongly induced by MeJA and UV-B radiation (Wang et al. 2007; Rice WRKY working group 2012). The overexpression of OsWRKY89 increased lignin and SA content and enhanced resistance to $M$. oryzae and tolerance to UV-B irradiation. Additionally, OsWRKY104 functioned downstream of ABA responsive element binding factor 1 (OsABF1) (Zhang et al. 2016). The overexpression of drought-inducible OsWRKY104 delayed the heading time by the suppression of Early heading date 1 (Ehd1) expression, suggesting that OsABF1 represses floral initiation at least partially through the activation of OsWRKY104 in response to water deficit (Zhang et al. 2016).

Knockout oswrky22 lines exhibited not only increased susceptibility to both virulent and avirulent $M$. oryzae strains but also altered cellular responses to nonhost M. grisea and the barley powdery mildew pathogen 
(Abbruscato et al. 2012). In addition, silencing OsWRKY22 enhanced sensitivity to aluminum stress ( $\mathrm{Li}$ et al. 2018). OsWRKY22 bound with the promoter of the OsFRDL4 (citrate transporter-like) gene via W-boxes and promoted $\mathrm{Al}$-induced citrate secretion and $\mathrm{Al}$ tolerance in rice (Li et al. 2018). OsWRKY45 participates in response to biotic and abiotic cues. OsWRKY45 can be phosphorylated in vitro by OsMPK4 and OsMPK6, which are activated via OsMKK4, OsMKK6 or OsMKK10-2, but not OsMKK1 (Ueno et al. 2013). The phosphorylation of OsWRKY45 via the OsMKK10-2-OsMPK6 cascade was required for its activity in SA-dependent disease resistance (Ueno et al. 2015). However, BTH-induced M. oryzae resistance was compromised by ABA treatment or abiotic stresses such as cold and high salinity, implying that the antagonistic effects of abiotic stresses possibly are mediated by ABA signaling. Two tyrosine phosphatases, OsPTP1/2, dephosphorylated OsMPK6 in the presence of ABA, leading to the deficient activation of OsWRKY45 and an increased susceptibility to the blast fungus (Ueno et al. 2015). Antagonistic effects were also observed among OsWRKY45-mediated responses to biotic stimuli. The overexpression of OsWRKY45 elevated resistance against biotrophic and hemibiotrophic pathogens such as the bacterium Xoo and the fungus M. oryzae, whereas knockdown of OsWRKY45 enhanced resistance to the necrotrophic fungal pathogen $R$. solani and herbivores such as the brown planthopper Nilaparvata lugens (Shimono et al. 2012; Huangfu et al. 2016).

\section{Concluding remarks}

WRKY TFs participate in a regulatory network that integrates internal and environmental factors to modulate various aspects of defense responses. The interactions among WRKY TFs provide insights into the dynamic regulation of target genes in a functionally cooperative and antagonistic manner. The auto- and crossregulation of the WRKY gene family imply that the WRKY cascade potentially fine-tunes this regulation by its own autoregulation. However, much effort is needed to resolve this mechanism in detail. Several WRKY TFs interact with receptors or components of the signal reception complex, suggesting a potential shortcut for signaling pathways. More importantly, the activation or enhanced activation of WRKY TFs is often associated with other signaling components, such as protein kinases. Therefore, how the $\mathrm{R}$ protein-WRKY complex recruits new members to promote plant immunity requires investigation. One particular strategy is to integrate the increasing amount of sequencing data with information on protein-protein and protein-DNA interactions in the WRKY-mediated regulation of important biological processes to establish a comprehensive WRKY signaling and transcriptional regulatory network. As WRKY TFs are essential for plant metabolism, clarifying how WRKY proteins regulate metabolism is a key step in modulating crop immunity. Additionally, it is worth noting that the versatility of WRKY TFs in defense against different pathogens, tolerance to abiotic stresses, and agronomic traits possibly limits the application of these genes to improve crops.

\section{Additional file}

Additional file 1: Table S1. WRKY family genes in 36 plantae species. (DOCX $27 \mathrm{~kb})$

\section{Abbreviations}

ABA: Abscisic acid; ALD1: AGD2-like defense response protein 1; ARF: AUXIN RESPONSE FACTOR; BTH: Benzothiadiazole; CGSNL: The committee on gene symbolization, nomenclature and linkage; ChIP-seq: Chromatin

immunoprecipitation-sequencing; CPK: Calcium-dependent protein kinase; CTWD: C-terminal WD; CYP: Cytochrome P450 monooxygenase; DAPseq: DNA affinity purification sequencing; Ehd1: Early heading date 1; EMSA: Electrophoretic mobility shift assay; ER: Endoplasmic reticulum; ETI: Effector-triggered immunity; flg22: 22-amino-acid epitope from flagellin; FMO1: Flavin-dependent monooxygenase 1; JA: Jasmonic acid; MEK or MKK: MPK kinase; MEKK: MPK kinase kinase; MPK: Mitogen-activated protein kinase; NTWD: N-terminal WD; PAMP: Pathogen-associated molecular pattern; Pip: Pipecolic acid; PTI: PAMP-triggered immunity; R: Resistance;

RBOH: Respiratory burst oxidase homolog; RLCK: Receptor-like cytoplasmic kinase; SA: Salicylic acid; SAR: Systemic acquired resistance; SLR/ IAA14: SOLITARY ROOT/INDOLE-3-ACETIC ACID14; SSB: Striped stem borer; TF: Transcription factor; TT7: Transparent testa 7; WD: WRKY domain; WGD: Whole genome duplication

\section{Acknowledgements}

We would like to acknowledge the anonymous reviewers for their work on this paper.

\section{Funding}

This work was supported by the Natural Science Foundation of China (31571947 and 31171833).

Availability of data and materials

Not applicable.

\section{Authors' contributions}

$X C$ and $Z G$ wrote the manuscript. $C L, H W$, and $Z G$ drew the figures. All authors read and approved the final manuscript.

Ethics approval and consent to participate

Not applicable.

\section{Consent for publication}

Not applicable.

\section{Competing interests}

The authors declare that they have no competing interests.

\section{Author details}

'Key Laboratory of Pest Monitoring and Green Management, MOA, China Agricultural University, Beijing 100193, China. ${ }^{2}$ Department of Plant Pathology, China Agricultural University, Beijing 100193, China. ${ }^{3}$ Joint Laboratory for International Cooperation in Crop Molecular Breeding, China Agricultural University, Beijing 100193, China. 
Received: 14 January 2019 Accepted: 28 March 2019 Published online: 16 April 2019

\section{References}

Abbruscato P, Nepusz T, Mizzi L, Del Corvo M, Morandini P, Fumasoni I, et al. OsWRKY22, a monocot WRKY22 gene, plays a role in the resistance response to blast. Mol Plant Pathol. 2012;13:828-41.

Adachi H, Nakano T, Miyagawa N, Ishihama N, Yoshioka M, Katou Y, et al. WRKY transcription factors phosphorylated by MAPK regulate a plant immune NADPH oxidase in Nicotiana benthamiana. Plant Cell. 2015;27:2645-63.

Ahuja I, Kissen R, Bones AM. Phytoalexins in defense against pathogens. Trends Plant Sci. 2012;17:73-90

Akagi A, Fukushima S, Okada K, Jiang CJ, Yoshida R, Nakayama A, et al. WRKY45 dependent priming of diterpenoid phytoalexin biosynthesis in rice and the role of cytokinin in triggering the reaction. Plant Mol Biol. 2014;86:171-83.

Alfieri M, Vaccaro MC, Cappetta E, Ambrosone A, De Tommasi N, Leone A. Coactivation of MEP-biosynthetic genes and accumulation of abietane diterpenes in Salvia sclarea by heterologous expression of WRKY and MYC2 transcription factors. Sci Rep. 2018;8:11009.

Babu MM, lyer LM, Balaji S, Aravind L. The natural history of the WRKY-GCM1 zinc fingers and the relationship between transcription factors and transposons. Nucleic Acids Res. 2006;34:6505-20.

Besseau S, Li J, Palva ET. WRKY54 and WRKY70 co-operate as negative regulators of leaf senescence in Arabidopsis thaliana. J Exp Bot. 2012;63:2667-79.

Birkenbihl RP, Kracher B, Somssich IE. Induced genome-wide binding of three Arabidopsis WRKY transcription factors during early MAMP-triggered immunity. Plant Cell. 2017;29:20-38.

Boudsocq M, Willmann MR, McCormack M, Lee $H$, Shan L, He $P$, et al. Differential innate immune signalling via $\mathrm{Ca}^{2+}$ sensor protein kinases. Nature. 2010;464:418-22.

Cai Y, Chen X, Xie K, Xing Q, Wu Y, Li J, et al. Dlf1, a WRKY transcription factor, is involved in the control of flowering time and plant height in rice. PLoS One. 2014;9:e102529.

Chang C, Yu D, Jiao J, Jing S, Schulze-Lefert P, Shen QH. Barley MLA immune receptors directly interfere with antagonistically acting transcription factors to initiate disease resistance signaling. Plant Cell. 2013;25:1158-73.

Chen F, Hu Y, Vannozzi A, Wu K, Cai H, Qin Y, et al. The WRKY transcription factor family in model plants and crops. Crit Rev Plant Sci. 2017a;36:311-35.

Chen H, Lai Z, Shi J, Xiao Y, Chen Z, Xu X. Roles of Arabidopsis WRKY18, WRKY40 and WRKY60 transcription factors in plant responses to abscisic acid and abiotic stress. BMC Plant Biol. 2010;10:281.

Chen J, Nolan TM, Ye H, Zhang M, Tong H, Xin P, et al. Arabidopsis WRKY46, WRKY54, and WRKY70 transcription factors are involved in brassinosteroidregulated plant growth and drought responses. Plant Cell. 2017b;29:1425-39.

Cheng H, Liu H, Deng Y, Xiao J, Li X, Wang S. The WRKY45-2 WRKY13 WRKY42 transcriptional regulatory cascade is required for rice resistance to fungal pathogen. Plant Physiol. 2015;167:1087-99.

Cheng X, Zhao Y, Jiang Q, Yang J, Zhao W, Taylor IA, et al. Structural basis of dimerization and dual W-box DNA recognition by rice WRKY domain. Nucl Acid Res. 2019. https://doi.org/10.1093/nar/gkz113.

Chi Y, Yang Y, Li G, Wang F, Fan B, Chen Z. Identification and characterization of a novel group of legume-specific, Golgi apparatus-localized WRKY and Exo70 proteins from soybean. J Exp Bot. 2015;66:3055-70.

Chi Y, Yang Y, Zhou Y, Zhou J, Fan B, Yu JQ, et al. Protein-protein interactions in the regulation of WRKY transcription factors. Mol Plant. 2013;6:287-300.

Cho $\mathrm{MH}$, Lee SW. Phenolic phytoalexins in rice: biological functions and biosynthesis. Int J Mol Sci. 2015;16:29120-33.

Chujo T, Miyamoto K, Ogawa S, Masuda Y, Shimizu T, Kishi-Kaboshi M, et al. Overexpression of phosphomimic mutated OsWRKY53 leads to enhanced blast resistance in rice. PLoS One. 2014;9:e98737.

Ciolkowski I, Wanke D, Birkenbihl RP, Somssich IE. Studies on DNA-binding selectivity of WRKY transcription factors lend structural clues into WRKYdomain function. Plant Mol Biol. 2008;68:81-92.

de Pater S, Greco V, Pham K, Memelink J, Kijne J. Characterization of a zincdependent transcriptional activator from Arabidopsis. Nucleic Acids Res. 1996;24:4624-31.

Deslandes L, Olivier J, Theulieres F, Hirsch J, Feng DX, Bittner-Eddy P, et al. Resistance to Ralstonia solanacearum in Arabidopsis thaliana is conferred by the recessive RRS1-R gene, a member of a novel family of resistance genes. Proc Natl Acad Sci U S A. 2002;99:2404-9.
Ding $M$, Chen J, Jiang Y, Lin L, Cao Y, Wang M, et al. Genome-wide investigation and transcriptome analysis of the WRKY gene family in Gossypium. Mol Gen Genomics. 2015a;290:151-71.

Ding ZJ, Yan JY, Li CX, Li GX, Wu YR, Zheng SJ. Transcription factor WRKY46 modulates the development of Arabidopsis lateral roots in osmotic/salt stress conditions via regulation of $A B A$ signaling and auxin homeostasis. Plant J. 2015b;84:56-69.

Duan MR, Nan J, Liang YH, Mao P, Lu L, Li L, et al. DNA binding mechanism revealed by high resolution crystal structure of Arabidopsis thaliana WRKY1 protein. Nucleic Acids Res. 2007;35:1145-54.

Fukushima S, Mori M, Sugano S, Takatsuji H. Transcription factor WRKY62 plays a role in pathogen defense and hypoxia-responsive gene expression in rice. Plant Cell Physiol. 2016;57:2541-51.

Gallego-Giraldo L, Shadle G, Shen H, Barros-Rios J, Fresquet Corrales S, Wang H, et al. Combining enhanced biomass density with reduced lignin level for improved forage quality. Plant Biotechnol J. 2016;14:895-904.

Gao X, Chen X, Lin W, Chen S, Lu D, Niu Y, et al. Bifurcation of Arabidopsis NLR immune signaling via $\mathrm{Ca}^{2+}$-dependent protein kinases. PLoS Pathog. 2013;9:e1003127.

Geilen K, Bohmer M. Dynamic subnuclear relocalization of WRKY40, a potential new mechanism of ABA-dependent transcription factor regulation. Plant Signal Behav. 2015;10:e1106659.

Grunewald W, De Smet I, Lewis DR, Lofke C, Jansen L, Goeminne G, et al. Transcription factor WRKY23 assists auxin distribution patterns during Arabidopsis root development through local control on flavonol biosynthesis. Proc Natl Acad Sci U S A. 2012;109:1554-9.

Grunewald W, Karimi M, Wieczorek K, Van de Cappelle E, Wischnitzki E, Grundler F, et al. A role for AtWRKY23 in feeding site establishment of plant-parasitic nematodes. Plant Physiol. 2008;148:358-68.

Guo P, Li Z, Huang P, Li B, Fang S, Chu J, et al. A tripartite amplification loop involving the transcription factor WRKY75, salicylic acid, and reactive oxygen species accelerates leaf senescence. Plant Cell. 2017;29:2854-70.

Han M, Kim CY, Lee J, Lee SK, Jeon JS. OsWRKY42 represses OsMT1d and induces reactive oxygen species and leaf senescence in rice. Mol Cells. 2014;37:532-9.

Hartmann M, Zeier T, Bernsdorff F, Reichel-Deland V, Kim D, Hohmann M, et al. Flavin monooxygenase-generated $\mathrm{N}$-hydroxypipecolic acid is a critical element of plant systemic immunity. Cell. 2018;173:456-69.

Hori K, Maruyama F, Fujisawa T, Togashi T, Yamamoto N, Seo M, et al. Klebsormidium flaccidum genome reveals primary factors for plant terrestrial adaptation. Nat Commun. 2014;5:3978.

Hu L, Wu Y, Wu D, Rao W, Guo J, Ma Y, et al. The coiled-coil and nucleotide binding domains of BROWN PLANTHOPPER RESISTANCE14 function in signaling and resistance against planthopper in rice. Plant Cell. 2017;29:3157-85.

Hu L, Ye M, Li R, Zhang T, Zhou G, Wang Q, et al. The rice transcription factor WRKY53 suppresses herbivore-induced defenses by acting as a negative feedback modulator of mitogen-activated protein kinase activity. Plant Physiol. 2015;169:2907-21.

Hu Y, Dong Q, Yu D. Arabidopsis WRKY46 coordinates with WRKY70 and WRKY55 in basal resistance against pathogen Pseudomonas syringae. Plant Sci. 2012; 185-186:288-97.

Huangfu J, Li J, Li R, Ye M, Kuai P, Zhang T, et al. The transcription factor OsWRKY45 negatively modulates the resistance of rice to the brown planthopper Nilaparvata lugens. Int J Mol Sci. 2016;17:697.

Hussain RMF, Sheikh AH, Haider I, Quareshy M, Linthorst HJM. Arabidopsis WRKY50 and TGA transcription factors synergistically activate expression of PR1. Front Plant Sci. 2018;9:930.

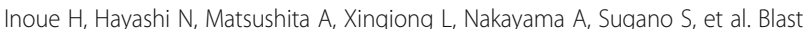
resistance of CC-NB-LRR protein Pb1 is mediated by WRKY45 through protein-protein interaction. Proc Natl Acad Sci U S A. 2013;110:9577-82.

Ishiguro S, Nakamura K. Characterization of a CDNA encoding a novel DNAbinding protein, SPF1, that recognizes SP8 sequences in the $5^{\prime}$ upstream regions of genes coding for sporamin and beta-amylase from sweet potato. Mol Gen Genet. 1994;244:563-71.

Ishihama N, Yamada R, Yoshioka M, Katou S, Yoshioka H. Phosphorylation of the Nicotiana benthamiana WRKY8 transcription factor by MAPK functions in the defense response. Plant Cell. 2011;23:1153-70.

Jalmi SK, Sinha AK. Functional involvement of a mitogen activated protein kinase module, OsMKK3-OsMPK7-OsWRK30 in mediating resistance against Xanthomonas oryzae in rice. Sci Rep. 2016;6:37974.

Jiang C, Shen QJ, Wang B, He B, Xiao S, Chen L, et al. Transcriptome analysis of WRKY gene family in Oryza officinalis Wall ex Watt and WRKY genes involved 
in responses to Xanthomonas oryzae pv. oryzae stress. PLoS One. 2017b;12: e0188742.

Jiang J, Ma S, Ye N, Jiang M, Cao J, Zhang J. WRKY transcription factors in plant responses to stresses. J Integr Plant Biol. 2017a;59:86-101.

Jones JD, Dangl JL. The plant immune system. Nature. 2006;444:323-9.

Kage U, Yogendra KN, Kushalappa AC. TaWRKY70 transcription factor in wheat QTL-2DL regulates downstream metabolite biosynthetic genes to resist Fusarium graminearum infection spread within spike. Sci Rep. 2017;7:42596.

Lal NK, Nagalakshmi U, Hurlburt NK, Flores R, Bak A, Sone P, et al. The receptor-like cytoplasmic kinase BIK1 localizes to the nucleus and regulates defense hormone expression during plant innate immunity. Cell Host Microbe. 2018;23:485-97.

Le Roux C, Huet G, Jauneau A, Camborde L, Tremousaygue D, Kraut A, et al. A receptor pair with an integrated decoy converts pathogen disabling of transcription factors to immunity. Cell. 2015;161:1074-88.

Lee H, Cha J, Choi C, Choi N, Ji HS, Park SR, et al. Rice WRKY11 plays a role in pathogen defense and drought tolerance. Rice. 2018;11:5.

Li G, Meng X, Wang R, Mao G, Han L, Liu Y, et al. Dual-level regulation of ACC synthase activity by MPK3/MPK6 cascade and its downstream WRKY transcription factor during ethylene induction in Arabidopsis. PLoS Genet. 2012;8:e1002767.

Li GZ, Wang ZQ, Yokosho K, Ding B, Fan W, Gong QQ, et al. Transcription factor WRKY22 promotes aluminum tolerance via activation of OsFRDL4 expression and enhancement of citrate secretion in rice (Oryza sativa). New Phytol. 2018;219:149-62.

Li J, Zhong R, Palva ET. WRKY70 and its homolog WRKY54 negatively modulate the cell wall-associated defenses to necrotrophic pathogens in Arabidopsis. PLoS One. 2017;12:e0183731.

Li R, Zhang J, Li J, Zhou G, Wang Q, Bian W, et al. Prioritizing plant defence over growth through WRKY regulation facilitates infestation by non-target herbivores. eLife. 2015;4:e04805.

Liang X, Chen X, Li C, Fan J, Guo Z. Metabolic and transcriptional alternations for defense by interfering OsWRKY62 and OsWRKY76 transcriptions in rice. Sci Rep. 2017;7:2474

Liu J, Chen X, Liang X, Zhou X, Yang F, Liu J, et al. Alternative splicing of rice WRKY62 and WRKY76 transcription factor genes in pathogen defense. Plant Physiol. 2016;171:1427-42.

Liu S, Kracher B, Ziegler J, Birkenbihl RP, Somssich IE. Negative regulation of ABA signaling by WRKY33 is critical for Arabidopsis immunity towards Botrytis cinerea 2100. eLife. 2015:4:e07295.

Liu ZQ, Yan L, Wu Z, Mei C, Lu K, Yu YT, et al. Cooperation of three WRKY-domain transcription factors WRKY18, WRKY40, and WRKY60 in repressing two ABAresponsive genes $A B / 4$ and $A B / 5$ in Arabidopsis. J Exp Bot. 2012;63:6371-92.

Ma Y, Guo H, Hua L, Martinez PP, Moschou PN, Cevik V, et al. Distinct modes of derepression of an Arabidopsis immune receptor complex by two different bacterial effectors. Proc Natl Acad Sci U S A. 2018;115:10218-27.

Machens F, Becker M, Umrath F, Hehl R. Identification of a novel type of WRKY transcription factor binding site in elicitor-responsive cis-sequences from Arabidopsis thaliana. Plant Mol Biol. 2014;84:371-85.

Maeo K, Hayashi S, Kojima-Suzuki H, Morikami A, Nakamura K. Role of conserved residues of the WRKY domain in the DNA-binding of tobacco WRKY family proteins. Biosci Biotechnol Biochem. 2001;65:2428-36.

Mao G, Meng X, Liu Y, Zheng Z, Chen Z, Zhang S. Phosphorylation of a WRKY transcription factor by two pathogen-responsive MAPKs drives phytoalexin biosynthesis in Arabidopsis. Plant Cell. 2011;23:1639-53.

Miao Y, Laun TM, Smykowski A, Zentgraf U. Arabidopsis MEKK1 can take a short cut: it can directly interact with senescence-related WRKY53 transcription factor on the protein level and can bind to its promoter. Plant Mol Biol. 2007;65:63-76

Mishra S, Triptahi V, Singh S, Phukan UJ, Gupta MM, Shanker K, et al. Wound induced tanscriptional regulation of benzylisoquinoline pathway and characterization of wound inducible PsWRKY transcription factor from Papaver somniferum. PLoS One. 2013;8:e52784.

Mohanta TK, Park YH, Bae H. Novel genomic and evolutionary insight of WRKY transcription factors in plant lineage. Sci Rep. 2016;6:37309.

Ning P, Liu C, Kang J, LV J. Genome-wide analysis of WRKY transcription factors in wheat (Triticum aestivum L.) and differential expression under water deficit condition. PeerJ. 2017;5:e3232.

Noutoshi Y, Ito T, Seki M, Nakashita H, Yoshida S, Marco Y, et al. A single amino acid insertion in the WRKY domain of the Arabidopsis TIR-NBSLRR-WRKY-type disease resistance protein SLH1 (sensitive to low humidity 1) causes activation of defense responses and hypersensitive cell death. Plant J. 2005:43:873-88.

O'Malley RC, Huang SC, Lewsey MG, Bartlett A, Nery JR, et al. Cistrome and epicistrome features shape the regulatory DNA landscape. Cell. 2016;165:1280-92

Oh SK, Baek KH, Park JM, Yi SY, Yu SH, Kamoun S, et al. Capsicum annuum WRKY protein CaWRKY1 is a negative regulator of pathogen defense. New Phytol. 2008; 177:977-89.

Papadopoulou K, Melton RE, Leggett M, Daniels MJ, Osbourn AE. Compromised disease resistance in saponin-deficient plants. Proc Natl Acad Sci U S A. 1999:96:12923-8.

Park CJ, Ronald PC. Cleavage and nuclear localization of the rice XA21 immune receptor. Nat Commun. 2012;3:920.

Peng $X$, Wang $H$, Jang JC, Xiao T, He H, Jiang D, et al. OsWRKY80-OsWRKY4 module as a positive regulatory circuit in rice resistance against Rhizoctonia solani. Rice. 2016;9:63.

Peng Y, Bartley LE, Chen X, Dardick C, Chern M, Ruan R, et al. OsWRKY62 is a negative regulator of basal and $X a 21$-mediated defense against Xanthomonas oryzae pv. oryzae in rice. Mol Plant. 2008;1:446-58.

Phukan UJ, Jeena GS, Shukla RK. WRKY transcription factors: molecular regulation and stress responses in plants. Front Plant Sci. 2016;7:760.

Qiu JL, Fiil BK, Petersen K, Nielsen HB, Botanga CJ, Thorgrimsen S, et al. Arabidopsis MAP kinase 4 regulates gene expression through transcription factor release in the nucleus. EMBO J. 2008;27:2214-21.

Rensing SA, Lang D, Zimmer AD, Terry A, Salamov A, Shapiro $H$, et al. The Physcomitrella genome reveals evolutionary insights into the conquest of land by plants. Science. 2008;319:64-9.

Rice WRKY working group. Nomenclature report on rice WRKY's-Conflict regarding gene names and its solution. Rice. 2012;5:3.

Rinerson Cl, Rabara RC, Tripathi P, Shen QJ, Rushton PJ. The evolution of WRKY transcription factors. BMC Plant Biol. 2015;15:66.

Rushton PJ, Macdonald H, Huttly AK, Lazarus CM, Hooley R. Members of a new family of DNA-binding proteins bind to a conserved cis-element in the promoters of alpha-Amy2 genes. Plant Mol Biol. 1995;29:691-702.

Sarris PF, Duxbury Z, Huh SU, Ma Y, Segonzac C, Sklenar J, et al. A plant immune receptor detects pathogen effectors that target WRKY transcription factors. Cell. 2015;161:1089-100.

Scarpeci TE, Zanor MI, Mueller-Roeber B, Valle EM. Overexpression of AtWRKY30 enhances abiotic stress tolerance during early growth stages in Arabidopsis thaliana. Plant Mol Biol. 2013;83:265-77.

Schluttenhofer C, Yuan L. Regulation of specialized metabolism by WRKY transcription factors. Plant Physiol. 2015;167:295-306.

Schön M, Toller A, Diezel C, Roth C, Westphal L, Wiermer M, et al. Analyses of wrky 18 wrky40 plants reveal critical roles of SA/EDS1 signaling and indoleglucosinolate biosynthesis for Golovinomyces orontii resistance and a loss-of resistance towards Pseudomonas syringae pv. tomato AvrRPS4. Mol PlantMicrobe Interact. 2013;26:758-67.

Sheikh AH, Eschen-Lippold L, Pecher P, Hoehenwarter W, Sinha AK, Scheel D, et al. Regulation of WRKY46 transcription factor function by mitogen-activated protein kinases in Arabidopsis thaliana. Front Plant Sci. 2016;7:61.

Shen H, Liu C, Zhang Y, Meng X, Zhou X, Chu C, et al. OsWRKY30 is activated by MAP kinases to confer drought tolerance in rice. Plant $\mathrm{Mol}$ Biol. 2012;80:241-53.

Shen QH, Saijo Y, Mauch S, Biskup C, Bieri S, Keller B, et al. Nuclear activity of MLA immune receptors links isolate-specific and basal disease-resistance responses. Science. 2007;315:1098-103.

Shimono M, Koga H, Akagi A, Hayashi N, Goto S, Sawada M, et al. Rice WRKY45 plays important roles in fungal and bacterial disease resistance. Mol Plant Pathol. 2012;13:83-94.

Shimono M, Sugano S, Nakayama A, Jiang CJ, Ono K, Toki S, et al. Rice WRKY45 plays a crucial role in benzothiadiazole-inducible blast resistance. Plant Cell. 2007:19:2064-76

Song $H$, Sun W, Yang G, Sun J. WRKY transcription factors in legumes. BMC Plant Biol. 2018;18:243

Souza CA, Li S, Lin AZ, Boutrot F, Grossmann G, Zipfel C, et al. Cellulose-derived oligomers act as damage-associated molecular patterns and trigger defenselike responses. Plant Physiol. 2017;173:2383-98.

Sun CX, Palmqvist S, Olsson H, Borén M, Ahlandsberg S, Jansson C. A novel WRKY transcription factor, SUSIBA2, participates in sugar signaling in barley by binding to the sugar-responsive elements of the iso 1 promoter. Plant Cell. 2003;15:2076-92. 
Suttipanta N, Pattanaik S, Kulshrestha M, Patra B, Singh SK, Yuan L. The transcription factor CrWRKY1 positively regulates the terpenoid indole alkaloid biosynthesis in Catharanthus roseus. Plant Physiol. 2011;157:2081-93.

Tian X, Li X, Zhou W, Ren Y, Wang Z, Liu Z, et al. Transcription factor OsWRKY53 positively regulates brassinosteroid signaling and plant architecture. Plant Physiol. 2017;175:1337-49.

Ueno Y, Yoshida R, Kishi-Kaboshi M, Matsushita A, Jiang CJ, Goto S, et al. MAP kinases phosphorylate rice WRKY45. Plant Signal Behav. 2013;8:e24510.

Ueno Y, Yoshida R, Kishi-Kaboshi M, Matsushita A, Jiang CJ, Goto S, et al. Abiotic stresses antagonize the rice defence pathway through the tyrosinedephosphorylation of OsMPK6. PLoS Pathog. 2015;11:e1005231.

van Verk MC, Pappaioannou D, Neeleman L, Bol JF, Linthorst HJ. A novel WRKY transcription factor is required for induction of PR-1a gene expression by salicylic acid and bacterial elicitors. Plant Physiol. 2008;146:1983-95.

VanEtten HD, Mansfield JW, Bailey JA, Farmer EE. Two classes of plant antibiotics: phytoalexins versus "phytoanticipins". Plant Cell. 1994;6:1191-2.

Veronese P, Nakagami H, Bluhm B, Abuqamar S, Chen X, Salmeron J, et al. The membrane-anchored BOTRYTIS-INDUCED KINASE1 plays distinct roles in Arabidopsis resistance to necrotrophic and biotrophic pathogens. Plant Cell. 2006;18:257-73

Wang H, Avci U, Nakashima J, Hahn MG, Chen F, Dixon RA. Mutation of WRKY transcription factors initiates pith secondary wall formation and increases stem biomass in dicotyledonous plants. Proc Natl Acad Sci U S A. 2010;107:22338-43.

Wang H, Hao J, Chen X, Hao Z, Wang X, Lou Y, et al. Overexpression of rice WRKY89 enhances ultraviolet $B$ tolerance and disease resistance in rice plants. Plant Mol Biol. 2007;65:799-815.

Wang H, Meng J, Peng X, Tang X, Zhou P, Xiang J, et al. Rice WRKY4 acts as a transcriptional activator mediating defense responses toward Rhizoctonia solani, the causing agent of rice sheath blight. Plant Mol Biol. 2015;89:157-71.

Wang J, Tao F, An F, Zou Y, Tian W, Chen X, et al. Wheat transcription factor TaWRKY70 is positively involved in high-temperature seedling plant resistance to Puccinia striiformis f. sp. tritici. Mol Plant Pathol. 2017;18:649-61.

Wang Y, Schuck S, Wu J, Yang P, Doring AC, Zeier J, et al. A MPK3/6-WRKY33ALD1-Pipecolic acid regulatory loop contributes to systemic acquired resistance. Plant Cell. 2018;30:2480-94.

Wu KL, Guo ZJ, Wang HH, Li J. The WRKY family of transcription factors in rice and Arabidopsis and their origins. DNA Res. 2005;12:9-26.

Xiao J, Cheng H, Li X, Xiao J, Xu C, Wang S. Rice WRKY13 regulates cross talk between abiotic and biotic stress signaling pathways by selective binding to different cis-elements. Plant Physiol. 2013;163:1868-82.

Xie K, Chen J, Wang Q, Yang Y. Direct phosphorylation and activation of a mitogen-activated protein kinase by a calcium-dependent protein kinase in rice. Plant Cell. 2014;26:3077-89.

Xie T, Chen C, Li C, Liu J, Liu C, He Y. Genome-wide investigation of WRKY gene family in pineapple: evolution and expression profiles during development and stress. BMC Genomics. 2018;19:490

Xie Z, Zhang ZL, Zou X, Huang J, Ruas P, Thompson D, et al. Annotations and functional analyses of the rice WRKY gene superfamily reveal positive and negative regulators of abscisic acid signaling in aleurone cells. Plant Physiol. 2005:137:176-89.

Xu H, Watanabe KA, Zhang L, Shen QJ. WRKY transcription factor genes in wild rice Oryza nivara. DNA Res. 2016;23:311-23.

Xu X, Chen C, Fan B, Chen Z. Physical and functional interactions between pathogen-induced Arabidopsis WRKY18, WRKY40, and WRKY60 transcription factors. Plant Cell. 2006;18:1310-26.

Yamasaki K, Kigawa T, Watanabe S, Inoue M, Yamasaki T, Seki M, et al. Structural basis for sequence-specific DNA recognition by an Arabidopsis WRKY transcription factor. J Biol Chem. 2012;287:7683-91.

Yan JY, Li CX, Sun L, Ren JY, Li GX, Ding ZJ, et al. A WRKY transcription factor regulates Fe translocation under Fe deficiency. Plant Physiol. 2016;171:2017-27.

Yang Y, Zhou Y, Chi Y, Fan B, Chen Z. Characterization of soybean WRKY gene family and identification of soybean WRKY genes that promote resistance to soybean cyst nematode. Sci Rep. 2017;7:17804.

Yao QY, Xia EH, Liu FH, Gao LZ. Genome-wide identification and comparative expression analysis reveal a rapid expansion and functional divergence of duplicated genes in the WRKY gene family of cabbage, Brassica oleracea var. capitata. Gene. 2015;557:35-42.

Yokotani N, Sato Y, Tanabe S, Chujo T, Shimizu T, Okada K, et al. WRKY76 is a rice transcriptional repressor playing opposite roles in blast disease resistance and cold stress tolerance. J Exp Bot. 2013;64:5085-97.
Zhang C, Liu J, Zhao T, Gomez A, Li C, Yu C, et al. A drought-inducible transcription factor delays reproductive timing in rice. Plant Physiol. 2016;171:334-43

Zhang L, Chen L, Yu D. Transcription factor WRKY75 interacts with DELLA proteins to affect flowering. Plant Physiol. 2018;176:790-803.

Zhang Y, Wang L. The WRKY transcription factor superfamily: its origin in eukaryotes and expansion in plants. BMC Evol Biol. 2005;5:1.

Zhou M, Lu Y, Bethke G, Harrison BT, Hatsugai N, Katagiri F, et al. WRKY70 prevents axenic activation of plant immunity by direct repression of SARD1. New Phytol. 2018;217:700-12.

Zhou QY, Tian AG, Zou HF, Xie ZM, Lei G, Huang J, et al. Soybean WRKY-type transcription factor genes, GmWRKY13, GmWRKY21, and GmWRKY54, confer differential tolerance to abiotic stresses in transgenic Arabidopsis plants. Plant Biotechnol J. 2008;6:486-503.
Ready to submit your research? Choose BMC and benefit from:

- fast, convenient online submission

- thorough peer review by experienced researchers in your field

- rapid publication on acceptance

- support for research data, including large and complex data types

- gold Open Access which fosters wider collaboration and increased citations

- maximum visibility for your research: over $100 \mathrm{M}$ website views per year

At BMC, research is always in progress.

Learn more biomedcentral.com/submissions 